Revue internationale P.M.E.

Économie et gestion de la petite et moyenne entreprise

\title{
Les dispositifs de gestion des compétences dans les PME : mythe ou réalité ? \\ Étude auprès de 28 dirrigeants de PME
}

\author{
Jocelyne Abraham, Franck Brillet, Patricia Coutelle et Annabelle Hulin
}

Volume 24, numéro 2, 2011

URI : https://id.erudit.org/iderudit/1012688ar

DOI : https://doi.org/10.7202/1012688ar

Aller au sommaire du numéro

Éditeur(s)

Presses de l’Université du Québec

ISSN

0776-5436 (imprimé)

1918-9699 (numérique)

Découvrir la revue

Citer cet article

Abraham, J., Brillet, F., Coutelle, P. \& Hulin, A. (2011). Les dispositifs de gestion des compétences dans les PME : mythe ou réalité ? Étude auprès de 28 dirigeants de PME. Revue internationale P.M.E., 24(2), 139-165.

https://doi.org/10.7202/1012688ar
Résumé de l'article

L'objectif de cet article est d'analyser la présence de dispositifs de gestion des compétences au sein des PME par la réalisation d'une étude empirique auprès de 28 entreprises de la Région Centre (France). Notre analyse s'inscrit dans le champ de recherches récentes comme celles s'intéressant à la gestion des compétences dans les TPE et les PME. Elle consistera, dans un premier temps, à identifier quels sont ces dispositifs et comment les classer pour, dans un second temps, en révéler les enjeux et les principaux enseignements. La problématique de cette recherche est donc de savoir quels sont les types de GEC (gestion des emplois et des compétences) mis en oeuvre au sein des PME, pour quelles raisons et comment elles procèdent. Pour répondre à cette problématique, une collecte de données primaires, par récits de pratiques, a été réalisée auprès de 28 dirigeants de PME de la Région Centre. L'analyse des données collectées laisse apparaître trois dimensions clés : les enjeux stratégiques, l'instrumentation et la transmission des connaissances. 


\title{
Les dispositifs de gestion des compétences dans les PME: mythe ou réalité? Étude auprès de 28 dirigeants de PME
}

Jocelyne ABRAHAM

IAE de Tours

Franck BRILLET

Université de Tours

Patricia COUTELLE

École polytechnique universitaire de Tours

Annabelle HULIN

Université de Tours

\section{MOTS CLÉS}

\section{PME - Gestion des emplois et des compétences -} Récits de pratiques - Dirigeant

\begin{abstract}
LES AUTEURS
JOCELYNE ABRAHAM est maître de conférences, habilitée à diriger des recherches. Elle est directrice de I'IAE de Tours. Elle est également directrice du master management des ressources humaines et stratégie de l'entreprise en formation continue. Membre du laboratoire de recherche CERMAT EA2109 de I'Université de Tours. Ses domaines de compétences sont la culture d'entreprise, la mobilité, le management stratégique des $\mathrm{RH}$ et l'attractivité et la rétention des talents. Elle est l'auteure de plusieurs articles et chapitres d'ouvrages et intervient en entreprises en matière de prévention des risques sociaux. Courriel: <jocelyne. abraham@univ-tours.fr>.

FRANCK BRILLET est maitre de conférences, habilité à diriger des recherches. II est directeur adjoint du laboratoire de recherche en management de l'Université de Tours (CERMAT EA2109), administrateur à l'Université de Tours et membre du conseil de gestion de la fondation Rabelais. II est également membre du Conseil national des universités. II a été directeur de l'IAE de Tours. Ses domaines de compétences sont la gestion des compétences, la prospective des métiers. Auteur d'articles et de chapitres d'ouvrages, il intervient également auprès d'organisations publiques et privées. Courriel: < franck.brillet@univ-tours.fr>.
\end{abstract}


PATRICIA COUTELLE est maître de conférences. Elle est actuellement directrice du master administration des entreprises-cycle École polytechnique universitaire de Tours. Elle a été également membre du Conseil national des universités et membre du jury du concours de l'agrégation d'économie et gestion. Ses domaines d'intervention sont relatifs au marketing, aux études et recherches, aux questions d'image. Auteure d'ouvrages relatifs à la démarche stratégique et marketing notamment en PME, elle intervient auprès de pôles de compétitivité et de PME. Courriel: <patricia.coutelle@univ-tours.fr>.

ANNABELLE HULIN détient un doctorat en sciences de gestion. Elle est membre du laboratoire de recherche CERMAT EA2109 de l'Université de Tours. Elle est spécialisée dans le transfert de compétences et la prospective des métiers. Elle s'intéresse également aux méthodologies d'analyses qualitatives assistées par ordinateur. Elle a écrit plusieurs articles pour la revue Management \& Avenir, la Revue des Sciences de Gestion, la Revue internationale de gestion et plusieurs chapitres d'ouvrages. Courriel: <annabelle.hulin@univ-tours.fr>.

\title{
RÉSUMÉ
}

L'objectif de cet article est d'analyser la présence de dispositifs de gestion des compétences au sein des PME par la réalisation d'une étude empirique auprès de 28 entreprises de la Région Centre (France). Notre analyse s'inscrit dans le champ de recherches récentes comme celles s'intéressant à la gestion des compétences dans les TPE et les PME. Elle consistera, dans un premier temps, à identifier quels sont ces dispositifs et comment les classer pour, dans un second temps, en révéler les enjeux et les principaux enseignements. La problématique de cette recherche est donc de savoir quels sont les types de GEC (gestion des emplois et des compétences) mis en œuvre au sein des PME, pour quelles raisons et comment elles procèdent. Pour répondre à cette problématique, une collecte de données primaires, par récits de pratiques, a été réalisée auprès de 28 dirigeants de PME de la Région Centre. L'analyse des données collectées laisse apparaître trois dimensions clés: les enjeux stratégiques, l'instrumentation et la transmission des connaissances.

\begin{abstract}
The aim of this paper is to analyze the presence of Management Skills Systems in SMEs, with the implementation of an empirical study conducted among $28 \mathrm{com}-$ panies of the Centre region (in France). Our analysis is part of recent researches on skill management in Very Small Businesses and Small and Medium Enterprises. It will consist, first, to identify what those systems are and how to classify them, and secondly, to reveal the main issues and draw the main lessons. The purpose of this research is to know what kind of Skills and Jobs Management is implemented in SMEs; for which reasons and how they proceed. To answer these questions, we collected data from 28 SMEs' managers, through stories of practices. Data analysis reveals three key dimensions: strategic issues, knowledge instrumentation and knowledge transmission.
\end{abstract}




\section{RESUMEN}

El objetivo de este articulo es de analizar la presencia de dispositivos de manejo de las capacidades en las PyMEs por medio de un estudio empirico con 28 empresas de la Región Centro (Francia). Nuestro análisis pertenece a un grupo de investigaciones recientes relacionadas con el manejo de las capacidades en las MPEs (Muy Pequeñas Empresas) y las PyMEs. Inicialmente el análisis se enfocara en identificar y clasificar estos dispositivos para luego dar a conocer los retos y las conclusiones principales. La problemática de esta investigación es de saber cuales tipos de MEC (Manejo de Empleos y de Capacidades) se implementan en las PyMEs, cuales son las razones de dichas implementaciones y como funcionan. Para resolver esta problemática, se recopilaron datos primarios, entrevistando a 28 gerentes de PyMEs de la Región Centro sobre sus practicas. El análisis de los datos recopilados permite identificar tres dimensiones claves: los retos estratégicos, el uso de instrumentos y la transmisión de los conocimientos.

\section{ZUSAMMENFASSUNG}

Der Artikel analysiert das Dispositiv zum Kompetenzmanagement bei KMU anhand einer empirischen Studie bei 28 Unternehmen aus der Region Centre in Frankreich. In einem ersten Schritt werden die Dispositive identifiziert und klassifiziert, um in einem zweiten Schritt die Herausforderungen darzustellen und Lehren daraus zu ziehen, $d . h$. welche Instrumente aus welchen Gründen wie verwendet werden? Anhand von Praxisberichten aller Unternehmensführer konnten drei wesentliche Dimensionen identifiziert werden: strategische Herausforderungen, Instrumentierung und Weitergabe von Fachkenntnissen.

\section{Introduction}

En matière de gestion des compétences, l'effet de mode est indéniable (Oiry, 2006). En France, on constate «une diffusion générale du discours sur la gestion des compétences » (Gilbert, 2003), mais il semble qu'aucune recette «miracle»n'existe à ce jour. On assiste également à une grande diversité d'intitulés lorsqu'on traite de ce sujet: gestion des compétences, gestion par les compétences, management des compétences, etc. (Dietrich,2008; Dietrich, Gilbert et Pigeyre, 2010). La littérature considère que la référence à l'une ou l'autre de ces terminologies atteste l'évolution même des dispositifs; c'est la raison pour laquelle elle considère que le management des compétences, ou par les compétences, est la forme la plus aboutie (Dietrich et al., 2010) de ces dispositifs. Notre étude s'intéressant aux dispositifs en général, nous adopterons le terme générique de «gestion des emplois et des compétences » pour qualifier ces derniers, n'étant pas en mesure de connaître leur degré d'évolution dans les entreprises concernées. 
Parler de gestion des emplois et des compétences (GEC), c'est aborder la question de la conception et de la mise en œuvre de dispositifs au sein des entreprises. Généralement, cette question est abordée uniquement au travers d'expériences de grandes entreprises qui illustrent la dimension instrumentale de tels dispositifs. Ainsi, on met souvent en perspective le recours à de nombreux outils de gestion essentiellement construits sur la base d'indicateurs quantitatifs et reposant sur un principe de rationalité instrumentale (Brillet et Hulin, 2010b; Brillet, Coutelle et Hulin, 2011), ce qui en constitue leur principale limite entraînant parfois un effet de désocialisation et de déconstruction des collectifs de travail au sein des entreprises. Ces études, principalement réalisées sur les grandes entreprises, pourraient autoriser à penser qu'elles seules sont concernées par la GEC; d'ailleurs, la loi de programmation pour la cohésion sociale de 2005 introduit, en France, l'obligation de négocier la mise en place d'un dispositif de gestion prévisionnelle des emplois et des compétences (GPEC) dans les entreprises d'au moins 300 salariés.

Dans ce contexte, l'objectif de cet article est d'attester la présence de tels dispositifs au sein des PME par la réalisation d'une étude empirique auprès de 28 entreprises de la Région Centre (France). Notre analyse s'inscrit dans le champ de recherches récentes comme celles s'intéressant à la gestion des compétences dans les TPE et les PME (Beaucourt et Schoenaers, 2010; Defélix et Retour, 2010; Parlier, 2010; Pacquelet, 2010). Elle consiste, dans un premier temps, à identifier quels sont ces dispositifs et comment les classer pour, dans un second temps, en révéler les enjeux et les principaux enseignements. La problématique de cette recherche est donc de savoir quels sont les types de GEC mis en œuvre au sein des PME, pour quelles raisons et de quelle manière elles procèdent.

Afin de répondre à cette problématique, nous présentons, dans la première section, un état des recherches en la matière. Si la GEC met du temps à s'inscrire dans les pratiques réelles de l'entreprise (Klarsfeld, 2003), il est donc important d'envisager plus globalement la problématique de la GRH au sein des PME (1.1) pour ensuite s'intéresser plus particulièrement aux dispositifs de GEC au sein de celles-ci (1.2). Cette revue de la littérature est suivie, dans une seconde section, de la présentation de l'enquête empirique et des résultats de cette enquête (2). Cette étude s'est attachée à adopter une méthodologie d'analyse qualitative réalisée auprès d'un échantillon de 28 dirigeants de PME (2.1). L'analyse a permis de présenter des résultats éclairant la problématique de l'existence de trois grandes catégories de dispositifs de GEC au sein des PME (2.2). 


\section{De la GRH aux dispositifs de GEC: état des lieux des recherches en PME}

Les dispositifs de GEC s'inscrivent plus globalement dans les pratiques de ressources humaines (RH) des entreprises. Avant de s'y intéresser plus précisément (1.2), il semble important de dresser un état des lieux sur la manière dont la GRH est considérée au sein des PME (1.1).

\subsection{La réalité de la GRH dans les PME}

C'est après avoir donné une définition de ce que l'on entend par PME (1.1.1) que nous abordons les questions de la considération de la GRH au sein de ces types d'entreprises (1.1.2).

\subsubsection{Le contexte de la PME}

Le choix d'une définition d'une PME a toujours été complexe et a donné lieu à de nombreux débats (D'Amboise et Muldowney, 1988). Si ces définitions sont multiples, elles sont néanmoins complémentaires et apportent toutes un regard pertinent à l'analyse de ce qu'est une PME. C'est donc en multipliant le recours à plusieurs de ces définitions que l'on peut mieux appréhender la réalité de ce qu'est une PME (Parlier, 2006, 2010).

Parmi ces différentes propositions, on peut se référer à celle adoptée par la Commission européenne en 2005 (tableau 1).

\section{TABLEAU 1}

\section{Définition des microentreprises et des petites et moyennes entreprises}

\begin{tabular}{lccc}
\hline \multicolumn{1}{c}{ Catégorie } & Effectif & Chiffre d'affaires (CA) & Total du bilan \\
\hline Moyenne entreprise & $<250$ & $\leq 50 \mathrm{M} €(1996-40 \mathrm{M})$ & $\leq 43 \mathrm{M} €(1996-27 \mathrm{M})$ \\
\hline Petite entreprise & $<50$ & $\leq 10 \mathrm{M} €(1996-07 \mathrm{M})$ & $\leq 10 \mathrm{M} €(1996-05 \mathrm{M})$ \\
\hline Microentreprise & $<10$ & $\leq 2 \mathrm{M} €(1996-n d)$ & $\leq 2 \mathrm{M} €(1996-n d)$ \\
\hline
\end{tabular}

Ainsi, les PME sont des entreprises de moins de 250 salariés, ayant un CA n'excédant pas 50 millions d'euros et 43 millions au total du bilan; trois critères quantitatifs sont ainsi déterminés. Cette définition peut être rapprochée d'autres qui apportent un regard légèrement différent, reposant sur des critères plus qualitatifs. À ce titre, Julien (1990) ajoute quatre critères complémentaires: la centralisation de la gestion, une faible spécialisation, des systèmes d'information interne et externe simples ou peu organisés et une 
stratégie intuitive ou peu formalisée. D'Amboise et Garand (1995) retiennent quatre spécificités de la PME: des pratiques de gestion moins développées et moins formalisées, une fonction ressources humaines fortement centralisée autour du propriétaire-dirigeant et des difficultés de délégation, peu de ressources financières matérielles et humaines et un manque global d'expertise dans les méthodes et techniques de GRH. Cette définition correspond à l'échantillon de notre étude où les dirigeants constituent le pivot central de la prise en compte de la gestion des problématiques RH. Torres (1998) indique que le critère de taille est insuffisant pour qualifier une entreprise de PME; son recours s'expliquant en partie par «commodité méthodologique». Il précise qu'une PME peut être assimilée à un modèle de proximité (Torres, 1997a, 1997b, 2000): proximité spatiale, proximité hiérarchique, proximité fonctionnelle et coordination de proximité.

Notre étude se réfère donc à une approche plurielle de la définition de la PME retenant les critères de la Commission européenne, mais également ceux plus qualitatifs des auteurs cités ci-dessus. Cette définition donnée, nous pouvons maintenant aborder la question de la GRH en PME.

\subsubsection{Les problématiques $R H$ des $P M E$}

D'une entreprise à l'autre, les besoins, les ressources, les difficultés et les enjeux RH ne sont pas du même ordre. Ils varient selon la taille de l'entreprise, mais également selon la fabrication de produits ou services différents et/ou de modes de production relevant de différentes logiques. L'activité ou les activités exercées par la PME, ainsi que l'utilisation de une ou plusieurs technologies, son emplacement géographique, l'intensité concurrentielle du secteur dans lequel elle se situe ou la dimension de ses marchés et leur nature, sont également des facteurs contingents importants pour comprendre la prise en compte de certaines problématiques RH au sein des PME.

Dans ce contexte, l'imbrication des structures juridiques, avec les règles économiques et sociales, les législations sociales et fiscales ne permettent pas toujours à une PME d'engager une réflexion de fond sur la GRH et de mettre en place un service RH aussi élaboré et structuré que celui retrouvé dans une grosse entité. Ces éléments ne permettent pas non plus de recourir à des instruments de GRH trop sophistiqués, trop coûteux et par ailleurs inadaptés aux conditions d'activité qui sont les leurs. Ces observations posent la question de la nécessité d'élaborer et de construire une véritable GRH-PME.

Il n'en demeure pas moins que le choix d'une politique de GRH en PME est avant tout affaire de jugement du dirigeant de l'entreprise. Et, 
lorsqu'on parle de dirigeant, on associe souvent cet élément à sa personnalité, elle-même déterminée par son état d'esprit, ses préjugés, ses représentations mentales, ses schémas cognitifs; bref, tous les éléments qui vont donner leur empreinte à la politique de ressources humaines qui sera mise en œuvre (Brillet,2000). Cette analyse justifie ainsi que l'échantillon de notre recherche soit composé de PME et que les acteurs interrogés soient les dirigeants.

Ainsi, en matière de $\mathrm{RH}$, les règles internes, les normes de comportement sont fortement orientées et déterminées par ce dirigeant à travers sa communication et l'information qu'il transmet (journaux internes, déclarations...), mais également par sa conception de la GRH, son comportement au travail, ses habitudes, etc. D'ailleurs, Fabi et Lacoursière (2008) montrent que les pratiques de GRH dans les PME se différencient en raison de facteurs tels que la personnalité du dirigeant, le style de leadership, le niveau de formation des employés, le marché du travail, les aspects légaux et culturels, etc. Paradas (2007) affirme que l'étude des croyances du dirigeant peut aider à comprendre le développement de certaines pratiques RH. La GRH est donc présente au sein des PME au même titre que les autres fonctions de l'entreprise et le propriétaire-dirigeant en contrôle la plupart des aspects et, pour quelques-unes, en y participant directement.

Contrairement aux idées reçues voulant que les dirigeants de PME s'intéressent plutôt aux problèmes de rentabilité financière, de trésorerie ou de recherche de nouveaux marchés, les problématiques de management et de personnel sont au cœur de leurs préoccupations. En raison de leur évolution, ces problématiques $\mathrm{RH}$ se révèlent de façon plus flagrante: croissance des effectifs, innovations technologiques et recherche de nouvelles compétences, adoption de nouveaux modes d'organisation du travail, transfert de compétences, renouvellement des populations, etc. Le développement des recherches en PME fait alors émerger de nouveaux champs d'analyse susceptibles d'enrichir les études en RH (Bayad, 1995; Bayad, Mahé de Boislandelle, Nebenhaus et Sarnin, 1995; Messeghem, Bories-Azeau et Noguera, 2009; Louart et Vilette, 2010).

Ces dernières années est apparu un domaine des RH en PME dans les recherches en GRH: celui des compétences. En effet, certains travaux révèlent les enjeux et les caractéristiques de la gestion des compétences dans les PME (Géniaux et Miara-Bonnardel, 2001; Defélix et Retour, 2003,2010; Parlier, 2006,2010), ce qui pose la question plus générale de l'importance des dispositifs de GEC au sein des PME. 


\subsection{Les recherches en PME sur les dispositifs de GEC}

En définitive, la littérature en sciences de gestion sur les problématiques de GRH en général (Beaucourt et Schoenaers, 2010) et de GEC, en particulier au sein des PME, est assez claire dans la mesure où ces études sont très peu nombreuses et ces problématiques sont jugées moins prioritaires par les dirigeants et les salariés des PME. En effet, les dirigeants manifestent plus volontiers leur intérêt pour des considérations techniques, technologiques, économiques et financières. Toutefois, assez récemment, on a vu se développer des ouvrages et des contributions concernant cette thématique (Louart et Vilette, 2010). Ce mouvement traduit le fait que le contexte d'évolution et de fonctionnement des PME est déterminant pour expliquer cette prise en compte des problématiques de GRH et de structuration des dispositifs de GEC. Les PME sont confrontées à de réels problèmes d'anticipation en matière de GRH; mettre en place de tels dispositifs devient donc une priorité pour elles. C'est pourquoi il convient de définir ce que l'on entend par dispositif de gestion (1.2.1) pour ensuite décrire les dispositifs de GEC mais également ceux qui ont intégré une dimension prévisionnelle (GPEC) dans leur contenu (1.2.2).

\subsubsection{Les dispositifs de gestion}

Globalement, un dispositif de gestion est ici considéré, au sens de De Vaujany (2006, p. 113), comme «un ensemble d'éléments de design organisationnel porté par une intention stratégique, produit et géré par le centre ou le pivot d'un collectif organisé, et qui vise à intégrer les outils et les acteurs de façon cohérente, et dans le respect de certaines règles de gestion». Les outils de gestion, qui composent ces dispositifs, sont, pour De Vaujany (2006, p. 113), dans un sens proche de celui donné par Moisdon (1997), «un ensemble d'objets de gestion intégrés de façon systématique et codifiée dans une logique fonctionnelle (ou toute autre logique d'acteur) et respectant un ensemble de règles de gestion» (cité par De Vaujany, 2006, p. 113).

Nous parlerons dans cette recherche de dispositif et d'instrument de GEC, plus que d'outil de GEC (Gilbert, 1997), considérant «la gestion des compétences comme un processus dans lequel la dynamique temporelle joue un rôle majeur» (Oiry, 2009, p. 3). 


\subsubsection{Des dispositifs de GEC aux dispositifs de GPEC}

Dans certains métiers et secteurs d'activité, les PME souffrent d'une rareté de compétences, elles ont des difficultés à être attractives en termes de rémunération et de perspectives de carrière, et cela les met en difficulté eu égard à leur possibilité de répondre aux exigences du marché ${ }^{1}$.

Ces points renforcent l'idée selon laquelle les dispositifs de GEC permettent, lorsqu'ils sont mis en place, de répondre aux nombreux enjeux révélés au sein des PME (Thamain, 2009). L'observation montre que, bien souvent, ces dispositifs s'inscrivent dans une certaine temporalité révélant ainsi une dimension prévisionnelle dans la gestion des effectifs, des emplois et des compétences. On parlera alors de dispositifs d'anticipation $\mathrm{RH}$. Au sein de ces dispositifs d'anticipation, les plus connus sont ceux de GPEC.

Le dispositif de GPEC peut s'apparenter à un processus de GRH complexe au cœur de la politique RH d'une organisation, mais façonné par le contexte dans lequel il prend forme, en l'occurrence celui de la PME (Gilbert, 1997). Ce processus de GRH peut ainsi être analysé de manière séquentielle, découpé en étapes, s'inscrivant dans un continuum temporel. La GPEC, c'est un ensemble d'outils dans sa partie visible, mais également une posture organisationnelle à la fois anticipative et responsabilisante, soit une réelle démarche centrée sur l'identification, l'analyse, le développement des compétences, l'anticipation et la sauvegarde des compétences clés.

Ce dispositif contient deux éléments clés, l'un se rapportant aux compétences et l'autre, aux emplois; chacun d'eux exige des démarches d'explicitation et des outils précis d'identification, de mesure, d'évaluation et de valorisation. Ainsi, faire de la GPEC requiert de l'entreprise:

- d'avoir mené une première réflexion de court et moyen terme autour de la GEC sur les éléments constitutifs des compétences en situation de travail et leur mesure (lors de l'évaluation notamment) tant sur les plans individuel que collectif;

1. À titre d'illustration, une recherche-action a été réalisée en 2010 par Brillet, Coutelle et Hulin auprès de PME d'un pôle de compétitivité. Ce pôle a fait le constat d'une problématique d'attractivité dont il fallait clarifier et identifier les causes, mais aussi d'une problématique de recrutement de la population d'ingénieurs. La recherche-action a consisté à produire un audit et recueil des besoins sur le recrutement, mais également à proposer des pistes de résolution de ces problématiques. 
- d'avoir mené une réflexion de plus long terme sur les effectifs et les emplois; une réflexion sur le contenu des emplois, mais également sur leur évolution (dimension prévisionnelle), le devenir des métiers (dimension prospective) (Brillet et al., 2011a) et la reconnaissance de la professionnalisation (Bureau et Igalens, 2008; Thamain, 2009).

De tels dispositifs d'anticipation nécessitent, néanmoins, la conception et l'utilisation de nombreux outils et la mobilisation de certains acteurs de l'organisation. Cela explique que plusieurs auteurs reprochent le caractère trop instrumental de tels dispositifs (Amadieu et Cadin,1996; Défélix, Dubois et Retour, 1997; Brillet et Hulin, 2010b; Brillet, Hulin et Martineau, 2011b). En outre, cela a parfois des conséquences importantes sur la crédibilité et la pertinence de ces dispositifs (Thamain, 2009), qui reposent bien souvent sur une seule dimension quantitative des effectifs et des emplois.

Cette analyse établit un lien possible entre la GEC et ce caractère d'anticipation des dispositifs mis en œuvre. Ainsi, l'analyse des données collectées, dans le cadre de cette recherche, s'attache à ce caractère d'anticipation et vérifie si ces liens sont avérés alors que certains pensent que les PME sont seulement préoccupées par le court terme et le quotidien.

\section{2. Étude empirique}

Deux points sont abordés: le premier présente le terrain de recherche choisi et la méthodologie mise en œuvre (2.1); le second expose les résultats de notre étude empirique et fait état de leur discussion (2.2).

\subsection{Méthodologie et structuration de l'échantillon}

Le processus de recherche mobilisé est abductif. Ainsi, son objectif «n'est pas réellement de produire des lois universelles, mais plutôt de proposer de nouvelles conceptualisations théoriques valides et robustes, rigoureusement élaborées» (Charreire et Durieux, 2004, p. 60). L'abduction nécessite test et discussion avant d'attribuer à la découverte un statut de loi comme dans la perspective de l'induction. Dès lors, la recherche effectuée a consisté en de nombreux allers-retours entre le terrain et la théorie.

Dans ce contexte, nous allons, dans un premier temps, expliciter la démarche de recherche mise en œuvre (2.1.1) pour, dans un second temps, préciser le terrain de recherche étudié (2.1.2). 


\subsubsection{Méthodologie de la recherche}

Après avoir précisé quels ont été les acteurs interrogés dans le cadre de cette recherche (2.1.1.1) et la méthode de collecte des données utilisée (2.1.1.2), nous présentons l'outil d'analyse des données qui a été choisi dans le cadre de cet article (2.1.1.3).

\subsubsection{Acteurs interrogés: les dirigeants de PME}

La méthodologie de recherche s'explique par l'observation du terrain. En effet, en participant à de nombreuses manifestations organisées par des réseaux professionnels tels que l'ANDRH, l'APM, la CGPME, le CJD et les $\mathrm{DCF}^{2}$, il nous est apparu que de nombreuses PME, d'après le témoignage de leur dirigeant, font de la GEC, pratiquent la GEC au quotidien et n'ont pas à «recevoir de leçons des grandes entreprises ». Partant de cela, il est donc devenu important de savoir, dans le cadre de cette recherche, ce que font réellement les PME lorsqu'il est question de GEC et lorsque leurs dirigeants affirment impulser une telle démarche dans leur organisation. C'est la raison pour laquelle l'échantillon de notre étude est composé de PME appartenant à au moins l'un de ces réseaux professionnels et de PME qui, selon les dires de leur dirigeant, ont adopté un dispositif de GEC.

D'un point de vue méthodologique, nous avons également considéré que le dirigeant serait l'interlocuteur privilégié de notre recherche. En effet, dans la majeure partie des PME, il n'existe pas de directeur des ressources humaines (DRH) et, bien souvent, c'est le dirigeant qui s'occupe des grandes problématiques liées à la politique de GRH de son entreprise. L'absence de DRH ne signifie pas qu'il y a absence de pratiques RH, dans la mesure où la GRH peut être partagée entre différents acteurs de l'entreprise (directeur administratif et financier, comptable, managers, etc.).

\subsubsection{Méthode de collecte des données: les récits de pratique}

La méthode des récits de pratique s'est naturellement imposée compte tenu du caractère exploratoire de l'étude (Baumard, Donada, Ibert et Xuereb, 2003) et de la complexité de l'objet étudié: les dispositifs de GEC.

La démarche que nous empruntons s'appuie sur la méthode biographique de recueil des données, ce qui nous permet de «décrire un phénomène dans toute sa complexité, selon une approche dite compréhensive, en prenant

2. ANDRH: Association nationale des directeurs des ressources humaines; APM: Association pour le management; CGPME: Confédération générale des petites et moyennes entreprises; CJD: Centre des jeunes dirigeants; DCF: Directeurs commerciaux de France. 
en compte un grand nombre de facteurs» (Giroux, 2003, p. 43). Au sein de cette approche biographique, nous mobilisons la méthode des récits de vie, qui est l'une des modalités possibles. À ce sujet, il faut remarquer que la mobilisation de cette méthode est encore rare au sein des recherches en sciences de gestion et, plus spécialement, au sein des recherches en GRH et en PME; seules 26 communications sont recensées entre 1990 et 2009 dans le cadre des congrès de l'AGRH ${ }^{3}$ et 13 , dans celui des congrès CIFEPME ${ }^{4}$ tenus entre 1993 et $2008^{5}$.

Bertaux (1997, p. 32) précise que l'on parle de récit de vie «dès lors qu'un sujet raconte à une autre personne, chercheur ou pas, un épisode quelconque de son expérience vécue». Il existe au sein des récits de vie trois grandes approches: les autoportraits, la compréhension des trajectoires sociales et les récits de pratique. Le dispositif de GEC étant considéré comme une pratique $\mathrm{RH}$, c'est dans cette dernière approche que nous nous inscrivons. Ainsi, chaque acteur interrogé «raconte sa propre "théorie" des événements survenus, dévoile son interprétation, met en scène sa vision par rapport à l'avenir, etc.» (Rouleau, 2003, p. 140). Là encore, l'intérêt de cette étude peut être souligné dans la mesure où peu d'auteurs ont recours à ces récits de pratique: trois communications sont recensées entre 1990 et 2009 lors des congrès de l'AGRH et aucune lors des congrès CIFEPME organisés entre 1993 et 2008.

Le guide de ce récit de pratique est synthétisé dans le tableau 2.

TABLEAU 2

Guide du récit de pratique

\begin{tabular}{|c|c|}
\hline Rubriques & $\begin{array}{l}\text { Questions et formulation exprimées } \\
\text { lors du récit de pratique }\end{array}$ \\
\hline Démarrage de l'entretien. & $\begin{array}{l}\text { «Racontez-nous votre expérience de la démarche } \\
\text { compétence dans votre entreprise...» }\end{array}$ \\
\hline $\begin{array}{l}\text { Relances éventuelles en cours } \\
\text { d'entretien si, et seulement si, } \\
\text { ces dimensions n'ont pas été } \\
\text { abordées. }\end{array}$ & $\begin{array}{l}\text { «Pouvez-vous nous parler de l'historique } \\
\text { de cette démarche?» } \\
\text { «Comment avez-vous fait?» } \\
\text { «Avec qui? } ? \text { « } \\
\text { «Pour quels effets?» }\end{array}$ \\
\hline
\end{tabular}

3. Association francophone de la gestion des ressources humaines.

4. Congrès international francophone sur l'entrepreneuriat et la PME.

5. Le recensement des communications a été effectué grâce aux sites Internet des associations académiques. 
L'utilisation des récits de pratique présente ici plusieurs avantages (tableau 3).

\section{TABLEAU 3}

\section{Avantages des récits de pratique pour cette étude}

1. Constituer une première étape de prise de connaissance avec le dirigeant interrogé.

2. Recueillir les interprétations, les représentations du dirigeant sur la pratique, et ce, au regard de l'expérience professionnelle antérieure de l'individu interrogé.

3. Prendre en compte l'importance du passé du dirigeant et des répercussions possibles sur l'interprétation des faits, de la pratique qu'il peut donner aux événements passés liés à la mise en œuvre de la pratique. Ces éléments antérieurs, importants dans l'interprétation qu'il se fait de la pratique, doivent être considérés comme une histoire réécrite.

4. Comprendre les situations actuelles liées à la pratique de GEC, mais également les projections à venir qu'il peut faire au regard de cette pratique (perspectives d'évolution, prévision, prévention, prospective).

\subsubsection{Outil d'analyse des données}

L'analyse des données collectées a été rendue possible grâce à l'utilisation d'un logiciel dédié à l'analyse des données qualitatives assistées par ordinateur (ADQAO): Alceste (Analyse des Lexèmes Co-occurrents d'un Ensemble de Segments de TExte). Ce dernier permet l'analyse lexicale de contenu. Elle correspond à une analyse des signifiants (Bardin, 2007). Elle permet de compter le nombre d'occurrences dans le corpus et d'analyser la composition du texte en termes de vocabulaire.

Ce logiciel est intéressant dans la mesure où, à partir d'un texte, il permet d'opérer une classification de ces données collectées à partir de mots et de groupes de mots (Naulleau, 2005). La méthode utilisée par Alceste est la classification descendante hiérarchique. Cette méthode procède par fractionnements successifs du texte: elle repère les oppositions les plus fortes entre les mots du texte et extrait ensuite des classes d'énoncés représentatifs (Rivière, 2009). Alceste recherche ensuite le vocabulaire caractéristique de chaque classe et repère les ressemblances et dissemblances de vocabulaire. Ce logiciel présente l'avantage d'offrir des pistes interprétatives.

\subsubsection{Structuration de l'échantillon de recherche: $28 \mathrm{PME}$}

Vingt-huit récits de pratique ont été réalisés auprès des dirigeants de PME de la Région Centre. Cette recherche a été soutenue par l'ARACT (Association régionale pour l'amélioration des conditions de travail) Centre (France). 
L'échantillon est présenté dans le tableau 4. La durée moyenne des récits de pratique oscille entre une heure et demie et trois heures. La collecte des données s'est effectuée entre septembre et décembre 2009. Afin de segmenter notre échantillon, nous avons été attentifs à trois caractéristiques des entreprises étudiées: le secteur d'activité, la taille de l'entreprise et la localisation de l'entreprise (département français). En effet, il s'agissait de vérifier si des différences existaient suivant la taille des PME, le secteur d'activité et, éventuellement, la localisation géographique. Certaines de ces PME appartiennent à plusieurs réseaux professionnels, mais nous avons fait figurer ici le réseau que le dirigeant considérait comme «principal».

\subsection{Présentation et discussion des résultats}

L'ensemble des données recueillies sur le terrain converge vers la même direction: l'existence d'une prise en compte de la GEC au sein des PME. Toutefois, nous rappelons que cette observation et les analyses qui sont présentées ne s'entendent qu'au regard de notre échantillon, qui peut ne pas être considéré comme représentatif des PME françaises.

À l'issue de l'analyse effectuée par le logiciel Alceste, trois classes ont été relevées et constituent différents types de dispositifs de GEC. Nous procédons pour chacun de ces types à la présentation des résultats puis à leur discussion.

\subsubsection{Classe 1- GEC stratégique: présentation et discussion des résultats}

Lors des récits de pratique, $24,70 \%$ du discours des dirigeants a été regroupé dans cette classe.

\subsubsection{Présentation générale des résultats}

Cette classe regroupe le discours des entreprises moyennes dont le secteur d'activité est plutôt orienté vers la construction, la plasturgie, la métallurgie, le BTP et l'immobilier. Les mots clés de cette classe sont: «besoin, démarche, compétences, ressources, définition, stratégie, vision, objectif, synergie». Ces termes mettent en évidence la compréhension de l'enjeu de la GEC dans ce type de PME qui est d'associer le type de dispositif à la stratégie du dirigeant. La dimension stratégique de la GEC est très présente dans le discours de ces dirigeants qui perçoivent dans ce dispositif un moyen d'atteindre leurs objectifs de moyen et long terme (3 à 5 ans). Les outils mobilisés sont orientés vers un référentiel des métiers et des compétences, un diagnostic et une cartographie des compétences et des métiers, outils que l'on peut qualifier d'assez 
TABLEAU 4

L'échantillon des entreprises interrogées

\begin{tabular}{|c|c|c|c|c|c|}
\hline $\begin{array}{c}\text { Codes } \\
\text { des } \\
\text { récits de } \\
\text { pratique }\end{array}$ & Effectifs & $\begin{array}{l}\text { Localisation } \\
\text { géographique }\end{array}$ & Activité & $\begin{array}{l}\text { Durée } \\
\text { du récit }\end{array}$ & Réseau \\
\hline $\mathrm{E} 1 * \mathrm{PE}$ & 35 & Indre-et-Loire & Informatique & $1 \mathrm{~h} 30$ & APM \\
\hline $\mathrm{E} 2 * \mathrm{ME}$ & 110 & Indre-et-Loire & Graphisme industriel & $1 \mathrm{~h} 45$ & CJD \\
\hline $\mathrm{E} 3 * \mathrm{ME}$ & 110 & Indre-et-Loire & Santé & $1 \mathrm{~h} 45$ & ANDRH \\
\hline $\mathrm{E} 4 * \mathrm{ME}$ & 83 & Indre-et-Loire & BTP & $2 \mathrm{~h}$ & ANDRH \\
\hline $\mathrm{E} 5 * \mathrm{ME}$ & 260 & Indre-et-Loire & Équipement & $2 \mathrm{~h}$ & CJD \\
\hline $\mathrm{E} 6 * \mathrm{ME}$ & 180 & Indre-et-Loire & Comptabilité & $3 \mathrm{~h}$ & CJD \\
\hline $\mathrm{E} 7 * \mathrm{ME}$ & 145 & Loir-et-Cher & Services à la personne & $1 \mathrm{~h} 30$ & CJD \\
\hline $\mathrm{E} 8 * \mathrm{PE}$ & 46 & Loir-et-Cher & Industrie & $2 \mathrm{~h}$ & APM \\
\hline $\mathrm{E} 9 * \mathrm{PE}$ & 22 & Loiret & Transport-logistique & $1 \mathrm{~h} 30$ & CJD \\
\hline $\mathrm{E} 10 * \mathrm{PE}$ & 12 & Loiret & Boulangerie & $1 \mathrm{~h} 30$ & CGPME \\
\hline $\mathrm{E} 11 * \mathrm{PE}$ & 19 & Indre-et-Loire & Restauration & $1 \mathrm{~h} 30$ & CGPME \\
\hline $\mathrm{E} 12 * \mathrm{ME}$ & 120 & Indre-et-Loire & Conception de jeux & $1 \mathrm{~h} 30$ & CJD \\
\hline $\mathrm{E} 13 * \mathrm{ME}$ & 220 & Loiret & Agroalimentaire & $2 \mathrm{~h}$ & $\mathrm{DCF}$ \\
\hline $\mathrm{E} 14 * \mathrm{ME}$ & 235 & Indre-et-Loire & Restauration & $1 \mathrm{~h} 30$ & ANDRH \\
\hline $\mathrm{E} 15 * \mathrm{PE}$ & 20 & Indre-et-Loire & Conseil & $1 \mathrm{~h} 30$ & DCF \\
\hline $\mathrm{E} 16 * \mathrm{ME}$ & 250 & Indre-et-Loire & Électronique & $1 \mathrm{~h} 30$ & CJD \\
\hline $\mathrm{E} 17 * \mathrm{ME}$ & 218 & Cher & Électroménager & $2 \mathrm{~h}$ & $\mathrm{DCF}$ \\
\hline $\mathrm{E} 18^{*} \mathrm{PE}$ & 30 & Indre-et-Loire & Hôtellerie-restauration & $1 \mathrm{~h} 30$ & APM \\
\hline $\mathrm{E} 19 * \mathrm{ME}$ & 240 & Indre-et-Loire & Construction & $3 \mathrm{~h}$ & CGPME \\
\hline $\mathrm{E} 20 * \mathrm{ME}$ & 140 & Loir-et-Cher & Chimie & $1 \mathrm{~h} 30$ & ANDRH \\
\hline $\mathrm{E} 21 * \mathrm{ME}$ & 123 & Loiret & Agroalimentaire & $1 \mathrm{~h} 30$ & CGPME \\
\hline $\mathrm{E} 22 * \mathrm{ME}$ & 180 & Indre-et-Loire & BTP & $1 \mathrm{~h} 30$ & ANDRH \\
\hline $\mathrm{E} 23 * \mathrm{ME}$ & 90 & Indre-et-Loire & Plasturgie & $1 \mathrm{~h} 45$ & ANDRH \\
\hline $\mathrm{E} 24 * \mathrm{PE}$ & 42 & Loiret & Textile & $2 \mathrm{~h}$ & $\mathrm{DCF}$ \\
\hline $\mathrm{E} 25^{*} \mathrm{ME}$ & 220 & Loiret & Immobilier & $3 \mathrm{~h}$ & APM \\
\hline $\mathrm{E} 26 * \mathrm{ME}$ & 180 & Indre-et-Loire & ВTP & $1 \mathrm{~h} 30$ & ANDRH \\
\hline $\mathrm{E} 27 * \mathrm{ME}$ & 10 & Indre-et-Loire & Travail temporaire & $1 \mathrm{~h} 45$ & ANDRH \\
\hline $\mathrm{E} 28 * \mathrm{ME}$ & 200 & Indre-et-Loire & Expertise comptable & $2 \mathrm{~h}$ & CGPME \\
\hline
\end{tabular}

Légende:PE:Petite entreprise (moins de 50 salariés). ME:Moyenne entreprise (plus de 50 salariés)

sophistiqués et «stratégiques» au regard, par exemple, de traditionnelles fiches de description d'emplois. Les acteurs identifiés comme importants sont le DRH, l'OPCA ${ }^{6}$, et les dirigeants dans la mise en œuvre de ce type de dispositif. Les principaux réseaux professionnels représentés dans cette

6. OPCA: Organisme paritaire collecteur agréé. 
classe sont le CJD et l'ANDRH. Les entreprises appartenant à cette classe sont essentiellement des entreprises où les effectifs sont assez importants; en effet, ces entreprises comptent en moyenne 170 salariés.

\subsubsection{Interprétation et discussion des résultats}

Les secteurs représentés dans cette classe sont principalement ceux qui sont soumis à de grandes évolutions et bouleversements, ce qui les a amenés à se poser des questions sur leur positionnement concurrentiel et leurs orientations stratégiques à venir. On remarque également que ces secteurs sont tous concernés par des problématiques d'attractivité et de fidélisation de leurs salariés. Toujours dans le prolongement de la prise en compte des acteurs dans le dispositif de GEC, on remarque la forte présence des réseaux professionnels tels que le CJD et l'ANDRH orientés sur des problématiques de ressources humaines essentiellement d'ordre stratégique. On peut, en effet, comprendre cette présence du CJD qui est bien en conformité avec des initiatives et discours développés autour de leur action; l'exemple du concours performance globale en est une excellente illustration. Par ailleurs, au regard de la taille, on remarque que ce sont essentiellement des ME. En effet, la préoccupation stratégique s'explique par la structure même de ces entreprises et la présence parfois de services responsables des ressources humaines travaillant en étroite collaboration avec les dirigeants. Ces derniers étant ainsi accompagnés par des professionnels des RH se consacrent plus aisément à une réflexion d'ordre stratégique pour trouver un alignement interne et externe entre les pratiques et la stratégie.

La GEC est appréhendée en tant que démarche mobilisant des acteurs. Ces acteurs jugés utiles à la conception et à la mise en œuvre de la démarche sont soit le dirigeant, soit le DRH lorsqu'il existe, soit l'OPCA. Cela révèle qu'un tel dispositif ne peut être conçu sans l'implication du dirigeant et sans une personne déterminée à porter ce projet:

«le chef d'entreprise doit se sentir engagé dans la démarche et même s'il délègue ce travail, il doit toujours se sentir concerné [...], car on est vraiment au cœur stratégique de l'entreprise» (E16*ME).

Ce dernier élément révèle le lien entre ce type de dispositif RH et la stratégie de la l'entreprise:

«il faut vraiment à mon sens que la gestion prévisionnelle des emplois et des compétences soit inscrite dans la stratégie politique de la structure» (E22* $\mathrm{ME})$; «je dois dire que c'est de mon rôle, car ce doit être en accord avec la stratégie de l'entreprise, ses objectifs et orientations» $(\mathrm{E} 23 * \mathrm{ME})$. 
Ce qui est également souligné, c'est la prise de conscience des dirigeants, dont le discours apparaît dans cette classe, sur l'importance stratégique de la GEC et sur sa spécificité:

«Dans le dispositif, ce que l'on sait bien faire en PME, c'est être proche des gens et de prévoir des mesures pour accompagner tout le monde et faire que cela se passe au mieux» (E21*ME).

L'ensemble de ces résultats va dans le sens de ceux de Thamain (2009, p. 283): «Si les grandes entreprises formalisent plus que les PME ou disposent de plus de moyens pour mettre en œuvre des outils de GPRH, les PME semblent plus innovantes suite à l'appropriation des dispositifs d'accompagnement en GPEC. Pour ces PME, la GPEC aide à formuler explicitement leur stratégie, connue dans les grands groupes.» Ce résultat est intéressant dans la mesure où il apporte une vision nouvelle du lien entre stratégie et GRH. En effet, traditionnellement, on présente la stratégie comme premier élément à formuler au sein des entreprises qui va ensuite être déployée dans les différentes fonctions de l'organisation. Or, nos résultats montrent que dans les PME la mise en œuvre d'un dispositif de GEC aide les PME à concevoir et formuler leur stratégie. Il s'agirait ici d'un soutien à la prise de conscience et à l'explicitation de leur stratégie.

On peut remarquer que, dans cette classe, les dirigeants expriment clairement la dimension prévisionnelle du dispositif considéré comme une réponse à des problématiques auxquelles ils se trouveront confrontés:

«ce qu'il faut bien comprendre, c'est de dire d'une entreprise qu'elle est dans une démarche prévisionnelle, c'est vraiment qu'on est avec une entreprise particulière et qui a bien compris que tout ce qui allait être lié à la formation continue des salariés est au cour de la stratégie des entreprises» (E23*ME).

En résumé, la mise en place de dispositifs de GEC est une démarche essentiellement proactive et préventive au regard de la vision que peuvent avoir les dirigeants de leur PME. Le dispositif, évoqué dans le discours de cette classe, s'inscrit dans une démarche d'anticipation des RH affirmant l'importance du caractère prévisionnel du dispositif. Cette vision intègre la GEC dans son environnement tant technique, technologique que concurrentiel. La GEC se structure ici autour d'une vision stratégique d'avenir.

\subsubsection{Classe 2 - Instrumentation de la GEC: présentation et discussion des résultats}

Lors des récits de pratique, $16,25 \%$ du discours des dirigeants a été regroupé dans cette classe. 


\subsubsection{Présentation générale des résultats}

Le discours classé ici concerne les dirigeants de PME plus petites issues principalement du secteur agroalimentaire et de la restauration. Les thèmes caractéristiques associés à cette classe sont: «entretien annuel, tableau de bord, indicateurs, évaluation, entretien, documents, fiches ». Ces thèmes renvoient à une vision plus opérationnelle de la GEC, une vision plutôt de court terme avec la création d'outils sans vision sur la finalité du dispositif. On retrouve ici le caractère très opérationnel de la GEC autour d'outils qui émergent en même temps qu'apparaissent des éléments contextuels et des contraintes (départ non prévu de salariés, recrutement non anticipé, volonté de s'approprier une technologie nouvelle, un savoir-faire particulier, etc.). Les principaux réseaux professionnels représentés dans cette classe sont l'APM, les DCF et la CGPME. Les entreprises appartenant à cette classe sont essentiellement des entreprises où les effectifs sont assez faibles; en effet, la taille moyenne des entreprises est de moins de 20 salariés.

\subsubsection{Interprétation et discussion des résultats}

Cette classe est assez représentative de secteurs d'activité où la dimension commerciale est forte, où l'intensité concurrentielle est également importante avec la présence sur ces secteurs de très grandes entreprises. Pour autant, ce sont des secteurs fragmentés où l'avantage concurrentiel recherché peut reposer sur le savoir-faire et la qualité du service rendu. Ainsi, les sources de différenciation étant multiples, il est indispensable de disposer d'outils opérationnels conçus pour répondre à des besoins de gestion des recrutements, des effectifs et des compétences. L'exemple de la restauration ou de l'agroalimentaire touche en effet des métiers de bouche aujourd'hui en tension; il est donc nécessaire d'attirer et de fidéliser son personnel en disposant d'outils flexibles, simples et pragmatiques. La particularité des entreprises présentes dans cette classe est d'être soumise à une forte cyclicité, ce qui explique la présence d'une dimension prévisionnelle dont nous reparlerons en synthèse. S'agissant de la taille, on remarque que les entreprises sont très petites (TPE/PE), ce qui permet de comprendre que le service responsable des problématiques de RH soit peu présent et partagé entre responsable administratif, comptable et managers. Ainsi, le dirigeant conçoit la GEC comme un «arsenal» d'outils au service des préoccupations commerciales assurant la gestion du quotidien et aidant les managers à faire face à leurs problématiques.

Le discours attribué à cette classe se caractérise par une approche très instrumentale de la GEC considérant le dispositif comme l'adjonction de nombreux petits outils, à relier les uns aux autres: 
«On a des grilles de compétences qui sont venues remplacer des fiches de postes qui n'étaient pas vraiment précises ni détaillées » (E18*PE); «à la fin de l'entretien annuel, je demande les souhaits d'évolution. J'analyse tous les entretiens annuels et à partir de là, je construis mon plan de formation» (E11*PE).

Ces différents points rejoignent les reproches de certains auteurs concernant le caractère trop instrumental des dispositifs de GPEC (Amadieu et Cadin, 1996; Defélix et al., 1997; Thamain, 2009). Il faut néanmoins convenir que généralement l'utilisation, la conception et l'application de tels outils rassurent les dirigeants:

«vous savez, on ne peut pas concevoir la GEC sans des outils et il en faut beaucoup, car on doit prévoir beaucoup de choses» (E10*ME); «moi, j'ai fait le point pour une GEC quels sont les outils dont on a besoin? Et je sais qu'on en aura jamais trop pour mieux gérer» (E18*PE).

Le risque peut également être lié aux situations où l'on va trouver un excès de formalisation avec le recours à des procédures très strictes et cet excès peut limiter le développement et l'autonomie des compétences outre de réduire considérablement la possibilité de souplesse et de flexibilité, ainsi que celle d'adaptation face aux évolutions de l'environnement.

En résumé, la GEC semble ici valorisée par les dirigeants par sa dimension technique et opérationnelle même si elle ne présente aucun caractère stratégique pour eux. Cette classe laisse apparaître que la GEC est envisagée de manière essentiellement réactive et défensive par rapport à différents évènements techniques, technologiques ou démographiques auxquels la PME a dû faire face. Pour l'essentiel, les dirigeants mettent en place, ou font mettre en place par leurs collaborateurs, des outils de GEC dès lors que des éléments de contexte l'imposent; il n'y a donc pas de vision et de dimension stratégique du dispositif à l'inverse de la première classe. Cela tend à démontrer que les outils nécessaires à la mise en place de dispositifs de GEC sont présents, mais on ne peut pas véritablement parler de dispositifs de gestion, cette vision instrumentale étant primordiale. En définitive, cette classe se caractérise par un fort développement des outils, mais un faible développement d'une vision globale du dispositif.

\subsubsection{Classe 3 - Transfert et génération: présentation et discussion des résultats}

Lors des récits de pratique, 59,05\% du discours des dirigeants a été regroupé dans cette classe. 


\subsubsection{Présentation générale des résultats}

Aucun secteur en particulier ne ressort de cette classe. La plupart des dirigeants interrogés sont représentés dans le discours de cette classe. Cette dernière porte à la fois sur une approche du transfert intraorganisationnel des compétences et sur une approche démographique et générationnelle. Les mots clés associés sont les suivants: «former, jeunes, tutorat, apprendre, professionnalisation, valoriser, évolution, pyramide des âges, lien, confiance, métier». L'idée principale du discours figurant dans cette classe est de pallier les problématiques de recrutement et de vieillissement des populations salariées.

L'apparition du terme «métier» est intéressante dans la mesure où elle envisage que le dispositif s'intéresse certes aux effectifs, aux emplois, aux compétences, mais également aux métiers et à leur évolution. Les principaux réseaux professionnels qui ressortent de cette classe sont le CJD et l'APM. La taille moyenne des entreprises de cette classe est de 90 salariés avec une amplitude allant de 11 à 180 .

\subsubsection{Interprétation et discussion des résultats}

La dimension prévisionnelle est essentielle dans cette classe: «d'ici cinq à dix ans, les métiers vont évoluer, et donc il faut travailler sur les compétences de nos salariés pour leur permettre de rester employables, que ce soit chez nous ou chez un autre, mais aussi par rapport aux besoins futurs des clients $(\mathrm{E} 11 * \mathrm{PE})$.

Par ailleurs, il apparaît que nous nous rapprochons dans cette classe de la définition de la GPEC donnée par l'ANACT ${ }^{7}$ : «la GPEC est une gestion anticipative et préventive des ressources humaines, fonction des contraintes de l'environnement et des choix stratégiques de l'entreprise», ce dont témoignent les propos de ce répondant: «avec ce que j'ai décidé pour l'entreprise, je veux une démarche qui m'y prépare et qui tienne compte des aléas liés à notre secteur» (E11*PE).

L'élément marquant dans l'analyse de cette classe reste celui de la prise de conscience de la structure de la pyramide des âges et de ses conséquences sur l'avenir de l'entreprise. Ainsi, la GPEC apparaît comme une recherche constante d'adéquation entre compétences détenues par les salariés et exigences des emplois, dans un futur plus ou moins proche (Boyer et Scouarnec, 2009; Brillet et Hulin, 2010a): «il y a une personne qui a une compétence particulière, qui est primordiale pour la société, il faut donc le connaître, l'iden-

7. Site Internet de l'ANACT: $<$ www.anact.fr/web/dossiers/travail-developpement-des-personnes/gpec $>$. 
tifier, et puis en fonction de la pyramide des âges, si cette personne va partir, si elle est bientôt à la retraite, il faut anticiper son remplacement, la formation de quelqu'un d'autre pour la remplacer» (E6*ME).

Les dirigeants expriment leurs préoccupations au regard de départs massifs à la retraite, mais également en termes de perte de compétences pour leur entreprise: «les effets attendus, je disais une moyenne d'âge qui baisse, le remplacement des professionnels qui partent à la retraite alors je vais le dire autrement, c'est dans l'entreprise, capter les compétences et les savoirs qui sont l'essence ou le cour de l'entreprise» (E8*PE).

Ce phénomène est d'autant plus ressenti en raison de la taille moyenne des entreprises qui, lorsqu'elles sont confrontées à des départs, constatent que cela peut concerner jusqu'à $35 \%$ de leur effectif dans les années à venir. En effet, il n'est pas rare d'observer des entreprises dont la taille moyenne est de 80 salariés et qui devront faire face au départ de plus de 20 collaborateurs dans les trois prochaines années.

Un autre élément intéressant est la prise de conscience que les départs des salariés peuvent ne pas être systématiquement liés à des départs à la retraite: «la démarche compétence est là pour améliorer leur professionnalisme, leur employabilité aussi, c'est-à-dire que le jour où ils veulent partir de l'entreprise, de s'être formés, d'avoir pris du temps là-dessus, ça leur permettra d'être plus qualifiés aux yeux d'une autre entreprise» (E1*PE).

En résumé, les PME qui apparaissent dans le discours de cette classe considèrent les dispositifs de GEC comme un atout majeur pour favoriser l'acquisition et la transmission de savoirs. Toutefois, nous constatons que la formalisation de la GEC au sein de ces PME est plutôt faible. L'un des premiers constats de l'analyse de cette classe est de mettre le transfert de compétences et la transmission au cœur des dispositifs de GEC, tant il est important pour elles d'acquérir une totale maîtrise des métiers par les salariés. Dans cette classe, la dimension collective et humaine est au cour des préoccupations des dirigeants interrogés, avec comme objectif sous-jacent de créer du lien social entre les générations en facilitant la cohésion et l'implication de tous. Ces éléments sont toutefois à rapprocher de l'appartenance à certains réseaux professionnels fortement présents dans cette classe (APM et CJD) qui véhiculent ces idées de recherche d'équilibre entre l'individu et le collectif, de l'importance de l'humain dans les entreprises tout en soulignant la nécessité d'instaurer un dialogue social constructif autour des pratiques de GRH avec toutes les parties prenantes (dont IRP et syndicats). 
Le discours de cette classe laisse également apparaître un élément intéressant dans la mesure où la transmission est au cœur des dispositifs de GEC (Brillet et Hulin, 2007). En effet, les dirigeants considèrent que les salariés doivent aider d'autres salariés pour qu'ils acquièrent une qualification et des compétences par et pour l'entreprise. Certaines situations montrent que la transmission est privilégiée en cas de succession entre un salarié arrivé à l'âge de la retraite et un salarié destiné à le remplacer (problématique de renouvellement des populations) alors que d'autres sont plus orientées vers des salariés qui sont nouvellement intégrés et qu'il faut former. L'analyse indique néanmoins qu'il faut être vigilant dans la mise en place de ces dispositifs de GEC car l'entreprise risque de voir apparaître une certaine compétition entre ceux qui transmettent et ceux qui reçoivent. De même, ce type d'orientation souligne l'importance de reconnaître ce processus de transmission dans les PME (rétribution matérielle ou immatérielle) (Brillet et Hulin, 2007; Hulin, 2010).

De manière synthétique, les analyses formulées pour ces différentes classes font apparaître trois catégories de dispositifs de GEC avec une prise en compte inégale de la dimension temporelle: parfois présente soit dans sa dimension prévisionnelle (classes 1,2 et 3), soit dans sa dimension prospective (classe 3). Dans une recherche de rapprochement et de synthèse des analyses, il est possible de proposer la construction d'un nouveau modèle de GPEC (figure 1) s'inspirant, entre autres, des travaux d'Ulrich (1997) et de Parlier (2006). Ce modèle retiendrait deux dimensions:

- la première porte sur les finalités principales de la GEC (axe horizontal), laissant apparaître une dimension technique et opérationnelle. Cette dimension s'apparente au système GRH, ce qui correspond à notre classe 2. De l'autre côté de l'axe, la GEC apparaît comme un mode de management porté par un dirigeant et/ou son encadrement; on retrouve ici nos classes 1 et 3 ;

- la seconde dimension (axe vertical) concerne les bénéficiaires de la GEC, selon que celle-ci ne bénéficie qu'à l'employeur (classe 1 de notre étude) ou qu'elle est «mutuellement profitable entre le salarié et l'entreprise» (Parlier, 2006, p. 3) marquant une nouvelle relation d'emploi (classes 2 et 3).

On peut alors positionner nos classes au regard de ce modèle (figure 1). 


\section{FIGURE 1}

\section{Les différents modèles de GEC}

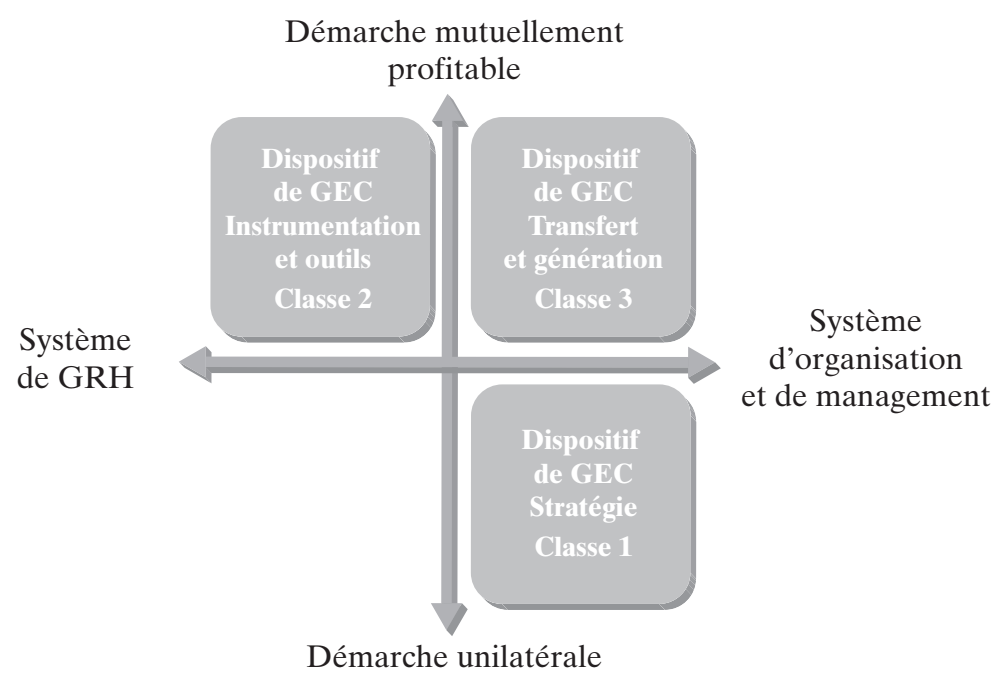

\section{Conclusion}

Grâce à cette recherche, nous pouvons confirmer l'existence de dispositifs de GEC au sein des PME. Les enjeux de la GEC sont pris en compte par l'ensemble de l'échantillon. S'intéresser à la GEC permet ainsi à ces dirigeants et à leur entreprise de faire face aux évolutions de l'environnement, de relever les défis de demain et donc d'assurer le développement et la pérennité de leur PME. La diversité des types observés permet néanmoins de constater que les motivations et les enjeux des PME peuvent expliquer l'adoption d'un type plutôt qu'un autre.

$\mathrm{Au}$ sujet des analyses et interprétations proposées, il convient de rappeler qu'elles reposent sur les perceptions et les représentations des dirigeants interrogés; une recherche nouvelle pourrait s'attarder à d'autres acteurs importants dans le dispositif tels que les salariés. Cette recherche souligne l'importance de la subjectivité des dirigeants pour comprendre les dispositifs de GEC tout en montrant la pluralité des dispositifs de GEC observés au sein des PME.

Développer et mettre en œuvre des dispositifs de GEC au sein des PME n'est pas impossible, mais cela doit conduire à dépasser les modèles réducteurs et normatifs que l'on voit émerger ici et là. Notre analyse tend égale- 
ment à montrer que lorsque des dispositifs de GEC sont issus de modèles normatifs et par nature réducteurs, ces derniers sont très peu performants, et ce, tant dans la réalisation des objectifs qu'on leur assigne et qui sont soumis à la contingence de leurs environnements (interne et externe) que dans l'efficience qu'ils présentent en termes d'optimisation des ressources et de minimisation de moyens.

On relèvera également que de tels dispositifs de GEC sont très peu actionnables dans les entreprises et encore moins au sein des PME de cette étude. Pour en faciliter la diffusion et l'adoption, il est important de s'appuyer sur l'expérimentation des dirigeants de PME. Leurs expériences, leur personnalité et leur histoire influent ainsi considérablement sur leur représentation des dispositifs de GEC, ce qui explique notre focalisation sur cet acteur dans le cadre de cette étude; ils sont en effet incontournables en raison de leur pouvoir décisionnel et des limites et leviers qu'ils mettent ou non à la politique de GRH au sein de leur entreprise.

Enfin, cette étude révèle qu'il existe deux catégories de dispositifs de GEC:

- formels, lorsque, par exemple, il y a des incitations fortes exprimées par le dirigeant ou certains partenaires (exemple de l'ARACT avec des incitatifs financiers et la proposition de formations adaptées et gratuites) ou des directives données par le dirigeant, la branche professionnelle ou la loi. La nature de ces incitations peut également provenir des contraintes que subissent les PME (contexte du bassin d'emploi, vieillissement de sa population, environnement technique et technologique, etc.);

- relationnels, lorsqu'on observe la présence de dispositifs de GEC afin de montrer l'exemple à d'autres, afin de se distinguer des autres PME du territoire ou bien simplement par imitation de ce que font les autres. Ce point n'est pas à écarter d'autant plus que toutes les PME de notre échantillon appartiennent à au moins un réseau professionnel. Certains de ces réseaux cherchant d'ailleurs, à travers leurs adhérents, à montrer leurs différences et leur capacité d'innovation par rapport à d'autres.

\section{Bibliographie}

AMADIEU, J.F. et L. CADIN (1996), Compétence et organisation qualifiante, Paris, Economica.

BARDin, L. (2007), L'analyse de contenu, Paris, Presses universitaires de France. 
BAUMARD, P., C. DONADA, J. IBERT et J.M. XUEREB (2003), «La collecte des données et la gestion de leurs sources », dans R.A. Thiétart, Méthodes de recherche en management, Paris, Dunod, p. 224-256.

BAYAD, M. (1995), «Éditorial. Dossier "La GRH dans les PME”», Revue Gestion 2000, vol. 11, n 1 , p. 91-94.

BAYAD, M., H. MAhÉ DE Boislandelle, D. NeBEnHAUS et P. SARNiN (1995), «Paradoxe et spécificités des problématiques de GRH en PME», Revue Gestion 2000, vol. 11, n ${ }^{\text {o } 1, ~ p . ~ 95-108 . ~}$

BEAUCOURT, C. et F. SCHOENAERS (2010), «Gestion des compétences en TPE Entreprises artisanales », dans P. Louart et M.A. Vilette, La GRH dans les PME, Paris, Vuibert, coll. «AGRH», p. 151-167.

BERTAUX, D. (1997), Les récits de vie, Paris, Nathan.

BOYER, L. et A. SCOUARNEC (2009), La prospective des métiers, Caen, Éditions EMS.

BRILlET, F. (2000), «Spécificités du management des rémunérations en contexte PME: l'exemple de l'intéressement», Revue de Gestion des Ressources Humaines, $\mathrm{n}^{\circ} 35$, avril.

BRILLET, F. et A. HULIN (2007), «Le tutorat outil polymorphe au service du transfert de connaissances, de compétences ? », Revue Sciences de Gestion, n 59, p. 91-135.

BRILLET, F. et A. HULIN (2010a), «Vers un renouvellement des dispositifs prévisionnels des ressources humaines: la question de la complémentarité entre prévision et prospective», Revue Management et Avenir, $\mathrm{n}^{\circ}$ 36, p. 245-262.

BRILlET, F. et A. HULIN (2010b), «Rationalité et "rationalités", dans les modèles d'anticipation des ressources humaines », dans F. Bournois et V. Chanut, Les organisations ont des raisons que la raison n'ignore pas... La rationalité managériale en recherches, Paris, Vuibert, juin, p. 241-250.

Brillet, F., COUTElle, P. et A. Hulin (2011a), «Les PME font-elles de la prospective des RH? », Revue Sciences de Gestion, n 79, p. 67-84.

BRILlet, F., Hulin, A. et R. MARTINEAU (2011 b), «Les dispositifs d'anticipation RH à l'épreuve de la rationalité...», Journée de recherche du LARGEPAParis 2, Paris, 15 mars.

BUREAU, S. et J. IGALENS (2008), «La professionnalisation: une nouvelle approche de la GPEC», dans F. Dupuich-Rabasse, Management et gestion des compétences, Paris, L'Harmattan, p. 31-54.

CHARREIRE, S. et F. DURIEUX (2004), «Explorer et tester: deux voies pour la recherche», dans R. A. Thiétart, Méthodes de recherches en management, Paris, Dunod, p. 57-81.

D’AmBOise, G. et D.J. GARAND (1995), «Identification des difficultés et des besoins des PME en matière de gestion des ressources humaines », Revue Gestion 2000, $\mathrm{n}^{\mathrm{o}} 1, \mathrm{p} .109-132$.

D’Amboise, G. et M. Muldowney (1988), «Management theory for small business : attempts and requirements », Academy of Management Journal, vol. 13, $\mathrm{n}^{\mathrm{o}} 2$, p. 22-240. 
DEFÉlix, C., M. Dubois et D. RETOUR (1997), «GPEC: une gestion prévisionnelle en crise», dans M. Tremblay et B. Sire, GRH face à la crise: GRH en crise?, Montréal, Presses de l'École des Hautes Études commerciales, p. 83-99.

DEFÉLIX, C. et D. RETOUR (2003), «Instrumenter la gestion des compétences: les PME aussi », dans A. KLARSFELD et E. OIRY, L'instrumentation de la gestion des compétences, Paris, Vuibert, coll. «AGRH».

DEFÉliX, C. et D. RETOUR (2010), «Gestion des compétences en PME de haute technologie», dans P. Louart et M.A. Vilette, La GRH dans les PME, Paris, Vuibert, coll. «AGRH», p. 169-173.

DE VAUJANY, F.X. (2006), «Pour une théorie de l'appropriation des outils de gestion: vers un dépassement de l'opposition conception-usage », Revue Management et Avenir, no 9, p. 109-126.

DiETRICH, A. (2008), Le management des compétences, Paris, Vuibert.

Dietrich, A., P. GilberT et F. PigeYRE (2010), Management des compétences. Enjeux, modèles et perspectives, $3^{\mathrm{e}}$ édition, Paris, Dunod.

FABI, B. et R. LACOURSIÈRE (2008), "Gestion des ressources humaines dans un contexte de PME», dans L.J. FILION, Management des PME. De la création à la croissance, Québec, Pearson Education, p. 231-243.

GÉNIAUX, I. et S. MIRA-BONNARDEL (2001), «La gestion des connaissances et des compétences dans les petites entreprises technologiques: une approche empirique», Revue internationale PME, vol. 14, n 2, p. 41-65.

GILBERT, P. (1997), L'instrumentation de gestion. La technologie de gestion, science humaine?, Paris, Economica.

GILBERT, P. (2003), «Jalons pour une histoire de la gestion des compétences », dans A. Klarsfeld et E. Oiry, Gérer les compétences: Des instruments aux processus, Paris, , Vuibert, coll. «AGRH», p. 11-31.

GirouX, N. (2003), «L'étude de cas», dans Y. Giordano, Conduire un projet de recherche: Une perspective qualitative, Caen, Éditions EMS, p. 41-84.

Hulin, A. (2010), Les pratiques de transmission du métier: de l'individu au collectif. Une application au compagnonnage, thèse de doctorat de l'Université de Tours, 27 avril.

JULIEN, P.A.(1990), «Vers une typologie multicritère des PME», Revue internationale $P M E$, vol. 3, ${ }^{\text {os }} 3-4$, p. 411-425.

JULIEN, P.A. (coord.) (1997), Les PME: bilan et perspectives, Paris, Economica.

KLARSFELD,A. (2003), «Rémunérer les compétences: analyse comparative et longitudinale de deux établissements du secteur du carton ondulé», dans C. Defélix, A. Klarsfeld et E. Oiry, Nouveaux regards sur la gestion des compétences, Paris, Vuibert, coll. «AGRH», p. 147-170.

LOUART, P. et M.A. VILETTE (2010), La gestion des ressources humaines dans les $P M E$, Paris, Vuibert, coll. «AGRH». 
Messeghem, K., I. BORIES-AZEAU et F. NOGUERA (2009), GRH, PME, transmission de nouvelles perspectives, Paris, Éditions Eyrolles.

MoISDON, J.C. (1997), Du mode d'existence des outils de gestion, Paris, Éditions Seli-Arslan.

NAUllEAU, M. (2005), «Rupture ou continuité des objets et dimensions de l'implication des salariés ayant vécu une période de restructuration? Intérêt exploratoire d'une perspective psychanalytique », $\mathrm{XVI}^{\mathrm{e}}$ Congrès de l'AGRH, Paris, actes électroniques.

OIRY, E. (2006), «La dynamique des instrumentations de gestion par les compétences - Proposition d'un cadre d'analyse», dans C. Defélix, A. Klarsfeld et E. Oiry, Nouveaux regards sur la gestion des compétences, Paris, Vuibert, coll. «AGRH», p. 13-31.

OIRY, E. (2009), «Un instrument de gestion des compétences peut-il connaître un second souffle?», XX ${ }^{\mathrm{e}}$ Congrès de l'AGRH, Toulouse, actes électroniques.

PACQUELET, C. (2010), «Gestion prévisionnelle des emplois et des compétences (GPEC) », dans P. Louart et M.A. Vilette, La GRH dans les PME, Paris, Vuibert, coll. «AGRH», p. 199-220.

PARADAS, A. (2007), «Le dirigeant comme levier de la RSE en TPE. Approche exploratoire basée sur l'utilisation des récits et d'une cartographie cognitive», Revue internationale PME, vol. 20, $\mathrm{n}^{\text {os }} 3-4$, p. 43-67.

PARLIER, M. (2006), «Gérer les compétences en PME», XVII ${ }^{\mathrm{e}}$ Congrès de l'AGRH, Reims, actes électroniques.

PARLIER, M. (2010), «Gérer les compétences en PME», dans P. Louart. et M.A. Vilette, La GRH dans les PME, Paris, Vuibert, coll. «AGRH», p. 175-198.

RIVIÈRE, A. (2009), Les effets des stratégies d'enrichissement de produits sur la valeur perçue d'un bien complexe, thèse de doctorat de l'Université de Tours.

RouleaU, L. (2003), «La méthode biographique», dans Y. Giordano, Conduire un projet de recherche. Une perspective qualitative, Caen, Éditions EMS, p. 133-172.

THAMAIN, J.L. (2009), «Anticiper en GRH, prévision et prospective, un diagnostic des pratiques et des représentations», Revue Management et Avenir, vol. 5, $\mathrm{n}^{\mathrm{o}} 25$, p. 272-289.

TORRES, O. (1997a), «Pour une approche contingente de la spécificité de la PME», Revue internationale PME, vol. 10, $\mathrm{n}^{\circ}$ 2, p. 9-43.

TORRES, O. (1997b), Pour une approche critique de la spécificité de gestion de la PME: application au cas de la globalisation, thèse de doctorat de l'Université de Montpellier 1.

TORRES, O. (1998), PME de nouvelles approches, Paris, Economica.

TORRES, O. (2000), «Du rôle et de l'importance de la proximité dans la spécificité de gestion des $\mathrm{PME}$ », $\mathrm{III}^{\mathrm{e}}$ Congrès international francophone sur les PME, actes électroniques.

UlRICH, D. (1997), Human Resource Champions: The Next Agenda for Adding Value and Delivering Results, Boston, Harvard Business School Press. 